\title{
Reliability of a repairable system
}

\author{
Li Wei ${ }^{a}$ and Wang Xiao \\ Department of Mathematics, Bohai University,Jinzhou, 121002, P. R. China \\ aliwei0627bhu@yeah.net.
}

Keywords: Redundant system, availability, up-time, circle-time.

\begin{abstract}
In this note, we study the reliability a repairable system by using the result of the positive unit eigenfunction of the eigenvalue 0 of the system operator is just steady-state solution of the system. We give the stationary availability and the mean up-time etc., of the complex standby system. Moreover, numerical results are provided to investigate the effects of various system parameters on the reliability indices.
\end{abstract}

\section{Introduction}

With the development of the modern technology and the world economy, the reliability problem of a system attracts more and more attentions. More and more people are working on the reliability of various systems [1]-[5]. In the same time, many valid methods have been developed to deal with the reliability for different systems. The repairable system is one of basic subjects in reliability. The availability and mean up-time are good evaluations of a repairable system performance, and occupy an increasingly important issues in power plant, manufacturing system and standby system. There is extensive literature on availability characteristics of repairable systems under various assumptions on the failures and repairs, see [6] and the references therein.

In most of these articles, methods used in the existing literature dealing with non-Markov systems involving many general random variables include the regenerative point technique (RPT) [6] and the supplementary variables method (SVM) [8]-[11]. In order to use the RPT, one has to correctly formulate and solve a system of Markov renewal equations, usually using an analytical method which is difficult for a non-Markov repairable system with only a few renewal points. By using the SVM, one can readily obtain all differential equations in terms of the state transition diagram of the model. However, it is still not easy to solve these differential equations because they usually involve some functions to be determined if there are at least two hazard rate parameters in one of the equations.

To overcome the above obstacles, we introduce a new method to calculate the formulas for reliability indices of a complex redundant system in the present paper. In 2009, W. L. Wang and G. Q. Xu investigated the well-posedness and stability of the redundant system in [12]. In 2012, F. Zheng and G. T. Zhu showed that the redundant system is exponentially stable by the bounded operators semigroups theory [13]. Based on these results, we discuss the steady-state reliability and show explicit expressions for the steady-state probabilities of the redundant system.

The rest of this paper is organized as follows. In section 2, some results on the well-posedness and stability of the system are reviewed. In section 3, we investigate the steady-state probabilities of the redundant system and obtain the explicit expressions for the stationary availability, mean uptime, mean down-time and mean circle-time. In section 4, several numerical simulation examples are provided to investigate the effects of various system parameters on the reliability indices. Conclusions are drawn in Section 5.

\section{Mathematical model formulation}

The redundant system discussed here comes from [12], which includes more information of the model. Let $a=\lambda_{0}+\lambda_{1}+\lambda_{2}+\lambda_{3}, p_{0}(t)$ be the probability that the system is in operable state at time $t, p_{1}(t)$ the probability that the system is in waiting state at time $t, p_{i}(t, x) d x$ the probability that the system 
is in operable state at time $t$ and elapsed repair time lies between $x$ and $x+d x$, where $i=2,3,4,5,6$, $p_{j}(t, x) d x$ the probability that the system is in degraded state at time $t$ and elapsed repair time lies between $x$ and $x+d \mathrm{x}$, where $j=8,9,10,11$, and $p_{i}(t, x) d x$ probability that the system is in failed state at time $t$ and elapsed repair time lies between $x$ and $x+d x$, where $i=7,12$. The governing equations of the system are as follows,

$$
\begin{aligned}
& {\left[\frac{d}{d t}+a\right] p_{0}(t)=\int_{0}^{\infty} p_{2}(t, x) \mu_{1}(x) d x+\int_{0}^{\infty} p_{3}(t, x) \mu_{2}(x) d x+\int_{0}^{\infty} p_{4}(t, x) \mu(x) d x} \\
& +\int_{0}^{\infty} \eta(x)\left[p_{5}(t, x)+p_{6}(t, x)\right] d x+\int_{0}^{\infty} p_{7}(t, x) \mu_{3}(x) d x+\int_{0}^{\infty} p_{12}(t, x) \beta(x) d x, \\
& {\left[\frac{d}{d t}+r_{1}\right] p_{1}(t)=\lambda_{0} \int_{0}^{\infty} p_{4}(t, x) d x, \quad\left[\frac{\partial}{\partial t}+\frac{\partial}{\partial x}+\mu_{3}(x)\right] p_{7}(t, x)=0} \\
& {\left[\frac{\partial}{\partial t}+\frac{\partial}{\partial x}+\mu_{1}(x)+\lambda_{2}\right] p_{2}(t, x)=0, \quad\left[\frac{\partial}{\partial t}+\frac{\partial}{\partial x}+\mu_{1}(x)+r_{2}\right] p_{8}(t, x)=0} \\
& {\left[\frac{\partial}{\partial t}+\frac{\partial}{\partial x}+\mu_{2}(x)+\lambda_{1}\right] p_{3}(t, x)=0, \quad\left[\frac{\partial}{\partial t}+\frac{\partial}{\partial x}+\mu_{2}(x)+r_{2}\right] p_{9}(t, x)=0} \\
& {\left[\frac{\partial}{\partial t}+\frac{\partial}{\partial x}+\mu(x)+\lambda_{0}\right] p_{4}(t, x)=0, \quad\left[\frac{\partial}{\partial t}+\frac{\partial}{\partial x}+\mu_{1}(x)+\lambda_{2}\right] p_{10}(t, x)=0} \\
& {\left[\frac{\partial}{\partial t}+\frac{\partial}{\partial x}+\eta(x)+\lambda_{1}+\lambda_{2}+r_{2}\right] p_{5}(t, x)=0,\left[\frac{\partial}{\partial t}+\frac{\partial}{\partial x}+\mu_{2}(x)+\lambda_{1}\right] p_{11}(t, x)=0} \\
& {\left[\frac{\partial}{\partial t}+\frac{\partial}{\partial x}+\eta(x)+\lambda_{1}+\lambda_{2}\right] p_{6}(t, x)=0, \quad\left[\frac{\partial}{\partial t}+\frac{\partial}{\partial x}+\beta(x)\right] p_{12}(t, x)=0}
\end{aligned}
$$

and with the boundary conditions

$$
\begin{aligned}
& p_{2}(t, 0)=\lambda_{1} p_{0}(t), p_{3}(t, 0)=\lambda_{2} p_{0}(t), p_{4}(t, 0)=\lambda_{2} \int_{0}^{\infty} p_{2}(t, x) d x+\lambda_{1} \int_{0}^{\infty} p_{3}(t, x) d x, \\
& p_{5}(t, 0)=\lambda_{0} p_{0}(t)+r_{1} p_{1}(t)+\int_{0}^{\infty} \mu_{1}(x) p_{8}(t, x) d x+\int_{0}^{\infty} \mu_{2}(x) p_{9}(t, x) d x, \\
& p_{6}(t, 0)=r_{2} \int_{0}^{\infty} p_{5}(t, x) d x+\int_{0}^{\infty} \mu_{1}(x) p_{10}(t, x) d x+\int_{0}^{\infty} \mu_{2}(x) p_{11}(t, x) d x, \\
& p_{7}(t, 0)=\lambda_{3} p_{0}(t), \quad p_{8}(t, 0)=\lambda_{1} \int_{0}^{\infty} p_{5}(t, x) d x, \quad p_{9}(t, 0)=\lambda_{2} p_{5}(t, x) d x \\
& p_{10}(t, 0)=\lambda_{1} \int_{0}^{\infty} p_{6}(t, x) d x+r_{2} \int_{0}^{\infty} p_{8}(t, x) d x, \\
& p_{11}^{\infty}(t, 0)=\lambda_{2} \int_{0}^{\infty} p_{6}(t, x) d x+r_{2} \int_{0}^{\infty} p_{9}(t, x) d x, \\
& p_{12}^{\infty}(t, 0)=\lambda_{2} \int_{0}^{\infty} p_{10}(t, x) d x+\lambda_{1} \int_{0}^{\infty} p_{11}(t, x) d x,
\end{aligned}
$$


and the initial conditions $p_{0}=1, p_{1}=p_{1}(0, x)=\cdots=p_{12}(0, x)=0$.

The equations (1)-(14) can be rewritten as an the abstract Cauchy problem in Banach space $X$

$$
(A C P) \quad\left\{\begin{array}{l}
\frac{d p(t)}{d t}=\mathcal{A} p(t) \\
p(0)=(1,0, \cdots, 0)^{T}
\end{array} \quad t \geq 0\right.
$$

in which $\mathcal{A}$ is defined in [12] and $X=\mathbb{R}^{2} \times\left(L^{1}\left(\mathbb{R}^{+}\right)\right)^{11}$ is Banach space. For $p=\left(p_{0}, p_{1}, p_{2}(x), \cdots, p_{12}(x)\right)$ $\in X$, the norm of $p$ is given by $\|p\|=\left|p_{0}\right|+\left|p_{1}\right|+\sum_{i=2}^{12} \int_{0}^{\infty}\left|p_{i}(x)\right| d x$.

Let $\mu_{4}(x)=\mu(x), \mu_{5}(x)=\beta(x)$ and $\mu_{6}(x)=\eta(x)$. The main results of the paper [12] are obtained under the following assumptions and they are also valid in this paper.

General Assumptions: There exist positive constants $H$ and $c$, such that for any $t \geq 0, \int_{t}^{\infty} e^{-\int_{t}^{u} \mu_{i}(u)} d u>$ $H$ and $c=\min \left\{\inf _{x \in \mathbb{R}^{+}} \mu_{i}(x), i=1, \cdots, 6\right\}$.

\section{Some steady-state reliability indices}

According to [5], $p_{0}(t)+\sum_{i=2}^{6} \int_{0}^{\infty} p_{i}(x, t) d x+\sum_{i=8}^{11} \int_{0}^{\infty} p_{1}(x, t) d x$ and $A V=\lim _{t \rightarrow \infty}\left(p_{0}(t)+\right.$ $\left.\sum_{i=2}^{6} \int_{0}^{\infty} p_{i}(x, t) d x+\sum_{i=8}^{11} \int_{0}^{\infty} p_{1}(x, t) d x\right)$ are the instantaneous availability and the stationary availability of the repairable system, respectively. The availability of the system is one of the most important reliability indices and engineers are especially interested in the steady-state availability. Now we will obtain the stationary availability of the system on the basis of the Theorem 1 and Theorem 2 of [12]. For convenience, set

$$
\begin{array}{lll}
f_{2}(x)=e^{-\int_{0}^{x} \mu_{1}(s)+\lambda_{2} d s} & f_{3}(x)=e^{-\int_{0}^{x} \mu_{2}(s)+\lambda_{1} d s} & f_{4}(x)=e^{-\int_{0}^{x} \mu(s)+\lambda_{0} d s} \\
f_{5}(x)=e^{-\int_{0}^{x} \eta(s)+\lambda_{1}+\lambda_{2}+r_{2} d s} & f_{6}(x)=e^{-\int_{0}^{x} \eta(s)+\lambda_{1}+\lambda_{2} d s} & f_{7}(x)=e^{-\int_{0}^{x} \mu_{3}(s) d s} \\
f_{8}(x)=e^{-\int_{0}^{x} \mu_{1}(s)+r_{2} d s} & f_{9}(x)=e^{-\int_{0}^{x} \mu_{2}(s)+r_{2} d s} & f_{12}(x)=e^{-\int_{0}^{x} \beta(s) d s} \\
a_{2}=\int_{0}^{\infty} \mu_{1}(x) f_{2}(x) d x & a_{3}=\int_{0}^{\infty} \mu_{2}(x) f_{3}(x) d x & a_{4}=\int_{0}^{\infty} \mu(x) f_{4}(x) d x \\
a_{5}=\int_{0}^{\infty} \eta(x) f_{5}(x) d x & a_{6}=\int_{0}^{\infty} \eta(x) f_{6}(x) d x & a_{8}=\int_{0}^{\infty} \mu_{1}(x) f_{8}(x) d x \\
a_{9}=\int_{0}^{\infty} \mu_{2}(x) f_{9}(x) d x & b_{j}=\int_{0}^{\infty} f_{j}(x) d x, & j=2,3, \cdots, 9,12
\end{array}
$$

Theorem 1. The stationary availability $A V$ of the system is

$$
A V=\frac{1+\sum_{i=4}^{6} b_{i} c_{i}+\sum_{i=8}^{9} b_{i} c_{i}+b_{2}\left(c_{10}+\lambda_{1}\right)+b_{3}\left(c_{11}+\lambda_{2}\right)}{1+c_{1}+\sum_{i=4}^{6} b_{i} c_{i}+\lambda_{3} b_{7}+\sum_{i=8}^{9} b_{i} c_{i}+b_{2}\left(c_{10}+\lambda_{1}\right)+b_{3}\left(c_{11}+\lambda_{2}\right)+b_{12} c_{12}}
$$

in which

$$
\begin{aligned}
& c_{1}=r_{1}^{-1} \lambda_{0} \lambda_{1} \lambda_{2} b_{4}\left(b_{2}+b_{3}\right), c_{4}=\lambda_{1} \lambda_{2}\left(b_{2}+b_{3}\right), \\
& c_{5}=\left[1-b_{5}\left(\lambda_{1} a_{8}+\lambda_{2} a_{9}\right)\right]^{-1}\left[\lambda_{0}+\lambda_{0} \lambda_{1} \lambda_{2} b_{4}\left(b_{2}+b_{3}\right)\right], \\
& c_{8}=\lambda_{1} b_{5}\left[1-b_{5}\left(\lambda_{1} a_{8}+\lambda_{2} a_{9}\right)\right]^{-1}\left[\lambda_{0}+\lambda_{0} \lambda_{1} \lambda_{2} b_{4}\left(b_{2}+b_{3}\right)\right], \\
& c_{9}=\lambda_{2} b_{5}\left[1-b_{5}\left(\lambda_{1} a_{8}+\lambda_{2} a_{9}\right)\right]^{-1}\left[\lambda_{0}+\lambda_{0} \lambda_{1} \lambda_{2} b_{4}\left(b_{2}+b_{3}\right)\right], \\
& c_{6}=r_{2}\left(b_{5} c_{5}+a_{2} b_{8} c_{8}+a_{3} b_{9} c_{9}\right)\left[1-b_{6}\left(\lambda_{1} a_{2}+\lambda_{2} a_{3}\right)\right]^{-1}, \\
& c_{10}=\lambda_{1} b_{6} c_{6}+r_{2} b_{8} c_{8}, c_{11}=\lambda_{2} b_{6} c_{6}+r_{2} b_{9} c_{9}, c_{12}=\lambda_{2} b_{2} c_{10}+\lambda_{1} b_{3} c_{11} .
\end{aligned}
$$

Let $p_{i}$ be the steady-state probability that the system is in state $i(i=0, \cdots, 12)$, Theorem 1 tells us $p_{0}=\|P\|^{-1}, p_{1}=c_{1}\|P\|^{-1}, p_{2}=\lambda_{1} b_{2}\|P\|^{-1}, p_{3}=\lambda_{2} b_{3}\|P\|^{-1}, p_{i}=c_{i} b_{i}\|P\|^{-1}(i=4,5,6)$, $p_{7}=\lambda_{3} b_{7}\|P\|^{-1}, p_{j}=c_{j} b_{j}\|P\|^{-1}(j=8,9), p_{10}=c_{10} b_{2}\|P\|^{-1}, p_{11}=c_{11} b_{3}\|P\|^{-1}$ and $p_{12}=$ $c_{12} b_{12}\|P\|^{-1}$. With these quantities in hands, we are able to obtain other reliability indices. 
Theorem 2. The mean up-time (MUP), mean down-time (MDP) and mean circle-time (MCP) of the redundant system are equal to

$$
\begin{gathered}
M U P=\frac{1+\sum_{i=4}^{6} b_{i} c_{i}+\sum_{i=8}^{9} b_{i} c_{i}+b_{2}\left(c_{10}+\lambda_{1}\right)+b_{3}\left(c_{11}+\lambda_{2}\right)}{\lambda_{0}+\lambda_{3}+\lambda_{2} b_{2} c_{10}+\lambda_{1} b_{3} c_{11}+r_{1} c_{4} b_{4}}, \\
M D P=\frac{c_{1}+b_{7} c_{7}+b_{12} c_{12}}{\lambda_{0}+\lambda_{3}+\lambda_{2} b_{2} c_{10}+\lambda_{1} b_{3} c_{11}+r_{1} c_{4} b_{4}}, \\
M C P=\frac{\|P\|}{\lambda_{0}+\lambda_{3}+\lambda_{2} b_{2} c_{10}+\lambda_{1} b_{3} c_{11}+r_{1} c_{4} b_{4}} .
\end{gathered}
$$

Proof: Let $M$ be the steady-state failure frequency of the system, then $M=\left(\lambda_{0}+\lambda_{3}\right) p_{0}+\lambda_{2} p_{10}+$ $\lambda_{1} p_{11}+r_{1} p_{4}$ by the definition of the steady-state failure frequency. However, the mean up-time (MUP), mean down-time (MDP) and mean circle-time (MCP) of the redundant system are $\frac{A V}{M}, \frac{1-A V}{M}$ and $\frac{1}{M}$, respectively. An easy computation yields these formulations. The proof of the Theorem 2 is complete.

\section{Numerical Example}

In the existing literature (e.g., see [9]-[11]), numerical results are often given under the assumption that the repair time distributions of the units are exponential, that is to say $\mu_{i}(x)$ are constants. It is not enough to show whether their methods are powerful or not since in this situation it is easy to obtain the reliability indices directly through simple computation. We shall carry out numerical simulations on more broad range. Some numerical results are first illustrated for the above models under the assumption that $\lambda_{0}=0.1, \lambda_{1}=0.2, \lambda_{2}=0.2, \lambda_{3}=0.01, r_{1}=0.05, r_{2}=0.05 ; \mu(x)=$ $\mu=0.3, \mu_{1}(x)=\mu_{1}=0.5, \mu_{2}(x)=\mu_{2}=0.6, \mu_{3}(x)=\mu_{3}=0.9, \eta(x)=\eta=0.7$ and

$$
\beta(x)= \begin{cases}0.1, & x \in[0,10] \\ s, & x \in(10, \infty) .\end{cases}
$$

The numerical results on the stationary availability, mean up-time, mean down-time and mean circletime are depicted in Fig.1-Fig.2.

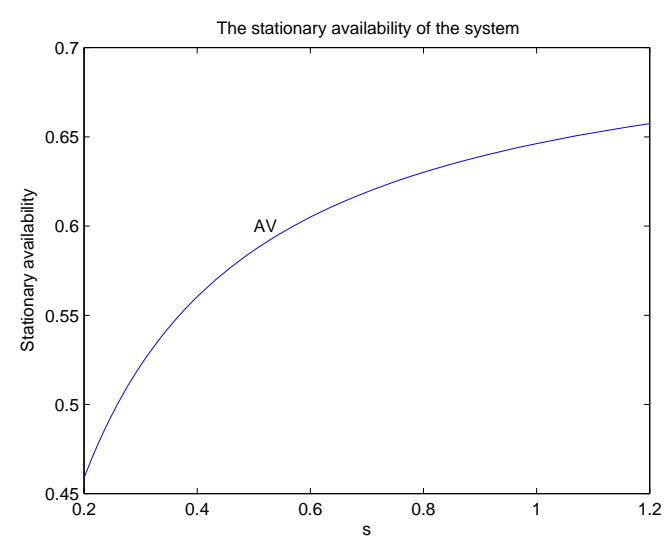

Fig. 1: The stationary availability $A V$ of the system

From the curves of Fig.1-Fig.2 we conclude that the stationary availability increases as $s$ increases. The increase is rapid initially and tends to vanish as $s$ becomes large. The mean circle-time and mean down-time decrease as $s$ increases. The decrease is rapid initially and tends to vanish as $s$ becomes large. However, the mean up-time are invariant as $s$ increases. 

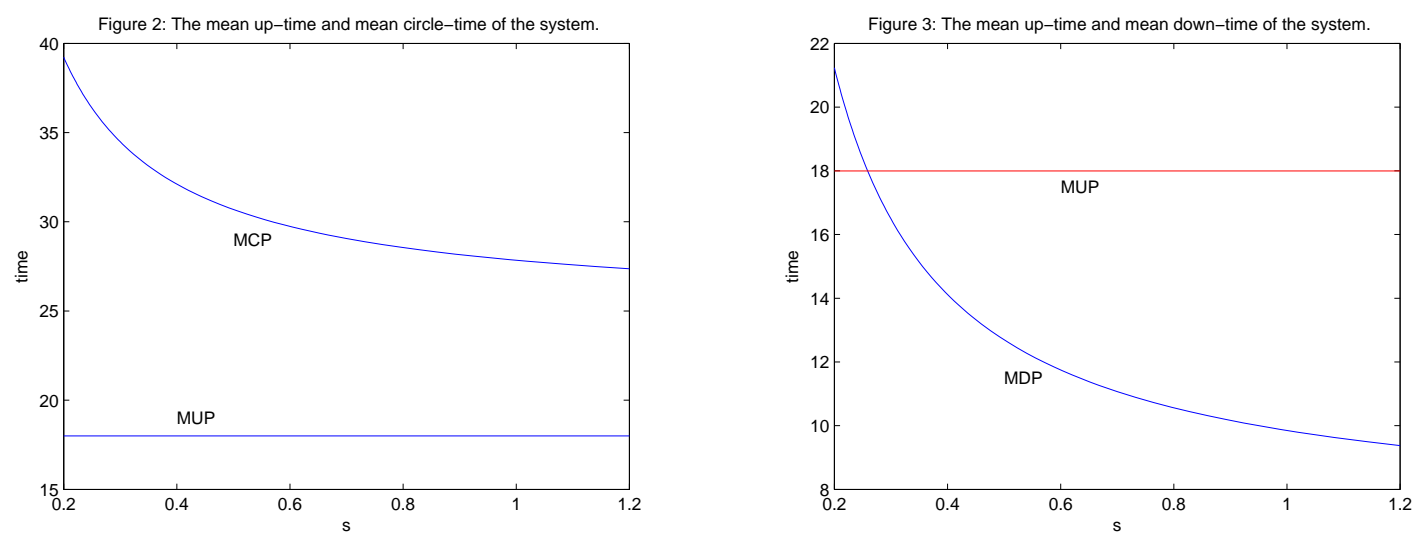

Fig. 2: The mean up-time, mean down-time and mean circle-time of the system

\section{Conclusion}

In this paper, we discussed a complex standby system with a method which is different from the traditional ones and obtained the explicit formulations of the steady-state availability of the system and the system's mean up-time, mean down-time and mean circle-time. Some numerical results were provided to show the effects of various system parameters on the steady-state availability and the mean time.

\section{Acknowledgment}

This work is partially supported by the National Science Natural Foundation in China (NSFC-11201037). The authors also gratefully acknowledge the helpful comments and suggestions of the reviewers, which have improved the presentation.

\section{References}

[1] Y. S. Juang, S. S. Lin, H. P. Kao, A knowledge management system for series-parallel availability optimization and design, Expert System Appl. , vol.34, pp.181-193, 2008.

[2] J. Cha, J. Choi, D. Park, J. Yoon, S. Moon, J. Watada and R. Billinton, Reliability evaluation for interconnection planning in north east Asia, International Journal of Innovative Computing, Information and Control, Vol.5, no.5, pp.1295-1312, 2009.

[3] S. Wang and J. Watada, Reliability optimization of a series-parallel system with fuzzy random life- times, International Journal of Innovative Computing, Information and Control, vol.5, no.6, pp.1547- 1558, 2009.

[4] W. C. Yeh, L. B. Cao and J. S. Jin, A Cellular Automata Hybyid Quasi-Random Monte Carlo Simulation for Estimating the one-to all Reliability of Acyclic Multi-State Information Networks, International Journal of Innovative Computing, Information and Control, vol.8, no.3, pp.20012014, 2012.

[5] J. H. Cao and K. Cheng, Introduction to Reliability Mathematics, Higher Eduation Press, Beijing, China, 2006.(in Chinese)

[6] S. B. Song, Y. L. Deng, Reliability analysis of a three-unit system in a changing environment, Microelectron. Reliab., vol.33, pp.637-640, 1993. 
[7] K.H. Wang and C.C. Kuo, Cost and probabilistic analysis of series systems with mixed standby components, Appl. Math. Modell., vol.20, pp.957-967, 2000.

[8] F. Zheng, G. Zhu, C. Gao. Well-pose-dness and stability of the repairable system with N failure modes and one standby unit. J. Math. Anal. Appl., vol.375, no.1, pp.174-184, 2011.

[9] L. M. Hu, D. Q. Yue and J. D. Liu, Probabilistic analysis of a series-parallel repairable system with three units and vacation, Appl. Math. Modell., vol.34, pp.2711-2721, 2009.

[10] L. Hu, J. Li and W. Fang, Reliability analysis of an N-component series system with M failure modes and vacation, ICIC Express Letters, vol.2, no.1, pp.53-58, 2008.

[11] L.M. Hu, J.D. L, Reliability analysis of a three-unit system with vacation and priority, ICIC Exp. Lett., vol.3, no.2, pp.171-176, 2009.

[12] W. L. Wang and G. Q. Xu, Stability analysis of a complex standby system with constant waiting and different repairman criteria incorporating environmental failure, Appl. Math. Modell., vol.33, pp.724-743, 2009.

[13] F. Zheng, G. T. Zhu, Exponential stability of a complex redundant system, Advance in Mathematics, vol.41, no.6, pp.713-722, 2012. 